\title{
Performances Des «Fermes Modèles » Du Corridor De Monkoto Dans La Province De La Tshuapa, République Démocratique Du Congo
}

\author{
Papy Bonkena Bokombola, PhD \\ Département d'économie agricole \\ Faculté des Sciences Agronomiques, Université de Kinshasa \\ République Démocratique du Congo \\ Alain Roddy Miteu Kalambayi, \\ Assistant, Département d'économie agricole \\ Faculté des Sciences Agronomiques, Université Pédagogique Nationale \\ République Démocratique du Congo
}

Doi:10.19044/esj.2020.v16n24p206 URL:http://dx.doi.org/10.19044/esj.2020.v16n24p206

\section{Résumé}

L'analyse des performances des « fermes modèles » constitue un enjeu majeur au regard de la lutte contre la pauvreté dans le contexte de la protection de la biodiversité dans le corridor de Monkoto. De façon générale, l'étude vise à analyser l'efficacité technique et la rentabilité économique des exploitations agricoles mixtes et intégrées développées dans le corridor de Monkoto, zone située entre les Blocs Nord et Sud du Parc National de Salonga. L'étude a employé l'approche centrée sur les entretiens individuels approfondis et les observations. Ces approches ont permis de collecter les données par la théorie de confrontation directe d'informations en vue de choisir les plus pertinentes pour cette étude. L'enquête a été conduite à l'aide du questionnaire individuel auprès de 10 fermiers modèles sur les 20 établis dans la zone. Les résultats montrent que le RNE (Revenu Net de l'Exploitant) moyen de 233.965,55 CDF et le score d'efficacité moyen de 0,1266 sont encore très faibles au regard du potentiel agro écologique que recèle la zone. Seulement 4 fermes modèles sur 10 sont rentables alors que la quasi-totalité des fermes sont inefficientes et n’opèrent de ce fait à leur niveau optimal. Toutefois, étant donné qu'elles sont dans un contexte des rendements d'échelle croissante, elles ont toutes une marge d'amélioration du volume de leur production avec le même volume de facteurs de production et par voie de conséquence, une marge de progression de la rentabilité. Ce qui peut favoriser la réplication et améliorer les conditions de vie des agriculteurs dans la zone et ainsi, baisser sensiblement la pression sur la biodiversité. 
Mots Clés : Fermes Modèles, Rentabilité, Protection De La Biodiversité, Corridor De Monkoto, République Démocratique Du Congo

\section{Performances Of « Model Farms » In The Monkoto Corridor In The Province Of Tshuapa, Democratic Republic Of Congo}

\section{Papy Bonkena Bokombola, PhD}

Département d'économie agricole Faculté des Sciences Agronomiques, Université de Kinshasa

République Démocratique du Congo

Alain Roddy Miteu Kalambayi,

Assistant, Département d'économie agricole

Faculté des Sciences Agronomiques, Université Pédagogique Nationale

République Démocratique du Congo

\section{Abstract}

Analyzing the performance of "model farms" is a major issue in the fight against poverty in the context of protecting biodiversity in the Monkoto corridor. Specifically, the study aims to analyze the technical efficiency and economic profitability of mixed and integrated farms developed in the Monkoto corridor, an area located between the North and South Blocks of Salonga National Park. The study used the focus approach on in-depth individual interviews and observations. This approach made it possible to collect data by the theory of direct confrontation of information in order to choose the most relevant for this study. The survey was conducted using the individual questionnaire among 10 model of the 20 farmers established in the area. The results show that the average NER (Net Income of the Operator) of CDF 233,965.55 and the average efficiency score of 0.1266 are still very low compared to the agro-ecological potential that the area contains. Only 4 out of 10 model farms are profitable while almost all of the farms are inefficient and therefore do not operate at their optimal level. However, given that they are in a context of increasing returns to scale, they all have room for improvement in the volume of their production with the same volume of production factors and consequently, room for improvement the profitability. This can encourage 
replication and improve the living conditions of farmers in the area and thus significantly reduce the pressure on biodiversity.

Keywords: Model Farms, Profitability, Biodiversity Protection, Monkoto Corridor, Democratic Republic Of Congo.

\section{Introduction}

Situé au cœur de la République Démocratique du Congo (RDC) et du Bassin du Congo, le Parc National de la Salonga (PNS) représente la plus grande étendue de forêt dense humide en Afrique et le deuxième plus grand Parc National de forêt tropicale dans le monde avec une biodiversité considérable. Le PNS et son paysage ${ }^{8}$ s'étendent sur une superficie de 102.847 $\mathrm{km}^{2}$ et abritent environ $90.000 \mathrm{~km}^{2}$ de forêt tropicale intacte. Cette forêt regorge une large diversité d'espèces animales et végétales et constitue un puits de carbone d'importance mondiale. Cette biodiversité est menacée principalement par la surexploitation de la faune à des fins commerciales (Union européenne, 2016), mais aussi par l'exploitation forestière, la pêche illégale et la pression agricole (agriculture itinérante sur brulis). Ceci entraine une diminution de la capacité de la forêt à rendre des services écosystémiques à la société (Hinojos, 2014) avec une baisse conséquente du rendement et de l'aptitude des communautés à répondre à leurs besoins de base (Ceroni et al., 2012).

Cette situation a suscité des inquiétudes à plusieurs échelles et amené à la prise des mesures de conservation de la biodiversité. En effet, dans son deuxième axe stratégique qui cadre avec la réduction des pressions exercées sur les habitats naturels, la stratégie nationale pour la préservation de la diversité biologique ${ }^{9}$ promet de favoriser la sédentarisation de l'agriculture et l'amélioration de la productivité des cultures (RDC, 2016). Dans cette logique, le développement de l'agriculture durable dans l'optique de développer des alternatives économiques fiables en vue de diminuer la pression sur la biodiversité et préserver l’intégrité, notamment du Parc National de la

${ }^{8}$ Le Paysage de la Salonga-Lukenie-Sankuru est reconnu par le Partenariat sur les Forêts du Bassin du Congo (PFBC) et la Commission des Forêts d'Afrique Centrale (COMIFAC).

${ }^{9}$ La stratégie nationale pour la diversité biologique est le résultat des consultations organises au niveau national et provincial (à travers toutes les provinces du pays). Elle intègre, de façon consensuelle, les préoccupations spécifiques des différentes provinces tant du point de vue de la conservation et de l'utilisation durable des ressources biologiques que celui de la mise en œuvre des programmes et des actions. 
Salonga, figure parmi les actions envisagées dans les communautés périphériques.

C'est ainsi que le modèle agricole durable «fermes modèles », associant les productions végétales et animales, a été développé dans le corridor de Monkoto depuis le début de l'année 2017. Ces fermes sont une innovation récente, introduite essentiellement à travers des projets de conservation et de développement agricole. Certains auteurs (Sneessens, 2014 ; Ruschawy et al., 2014) estiment que ce système de production («polyculture-élevage ») offre des avantages économiques et environnementaux supposés répondre à une partie de crises environnementales attribuées à l'agriculture spécialisée. Vue comme une alternative prometteuse à la spécialisation (Russelle et al., 2007 ; Wilkins, 2008), ce type d'exploitation, selon les niveaux d'intégration entre les ateliers d'élevage et de cultures, est toutefois plus ou moins à même de répondre aux défis actuels de la durabilité de l’agriculture en périphérie de Salonga. Dans cette approche, le développement des «fermes modèles » et leur diffusion dans le contexte du PNS pourrait trouver son sens.

Cependant, jusqu’à présent, ces initiatives n’ont pas encore été évaluées tant sur le plan technique, social, économique qu’environnemental. Connaitre le niveau de performance de la production au niveau des « fermes modèles pilotes » paraît utile pour les autres agriculteurs. Ceci se justifie par le fait que l'adoption de ce genre d'innovation, nécessite des exemples de réussite concrets d'une part et des politiques qui permettront d'assurer la viabilité et la durabilité de ces exploitations mixtes, surtout dans le contexte de conservation de la biodiversité d'autre part.

Face à cette préoccupation, cette étude vise à analyser la performance des «fermes modèles » en vue de dégager des mesures appropriées d'amélioration de l'approche et de diffusion du modèle agricole. Plus spécifiquement, l'étude analyse premièrement l'efficacité technique et deuxièmement la rentabilité économique des exploitations agricoles mixtes et intégrées développées dans le corridor de Monkoto.

\section{Cadre théorique de référence}

Selon le Fonds Mondial pour la Nature les « fermes modèles » sont des systèmes de production dans lesquels on retrouve de façon intégrée et diversifiée la production animale et végétale avec une introduction progressive des systèmes agroforestiers (WWF, 2018). Ainsi, toutes les fermes de la zone d'étude pratiquent l'association cultures et élevage sur une même exploitation. Elles correspondent à des systèmes d'exploitation " polyculture-élevage » ou « systèmes mixtes », ( « mixed crop-livestock systems ») (Gibon et al., 2011) ou encore «systèmes intégrés » («integrated crop-livestock systems ») (Hendrickson et al., 2008), en référence au niveau d’intégration entre 
l'élevage et les cultures. Seré et al. (1996) définissent les exploitations de polyculture-élevage en considérant les dimensions agronomiques et économiques : «des systèmes d'élevage dans lesquels au moins $10 \%$ de la matière sèche pour l'alimentation animale provient de coproduits végétaux de l'exploitation, ou plus de $10 \%$ de la valeur des produits provient d'activités agricoles non liées à l'élevage ».

Le cadre opératoire choisi pour conduire cette étude repose sur les deux dimensions : la rentabilité économique et l'efficacité technique. Sempore et al. (2015) montrent que ces indicateurs de performance constituent des principales règles de décision des fermiers étant donné qu'ils permettent de représenter de façon plus flexible le fonctionnement de l'exploitation et d'optimiser les systèmes agricoles.

La performance économique a été abordée sous l'angle de la rentabilité des fermes modèles pilotes. Etant donné que les fermes exploitent plusieurs spéculations, l'analyse de la rentabilité économique des fermes s'est inscrite dans la logique des travaux de Riollet (2011). Selon cet auteur, dans un contexte d'absence des données, il faut considérer l'entreprise agricole (la ferme modèle pilote) comme un tout en ce sens qu'en polyculture et associations des cultures ayant des cycles végétatifs différents, il n’est pas aisé d'établir une clé de répartition des charges tant opérationnelles que structurelles. Etant donné le système de culture encore traditionnel, Levallois et al. (2014) recommandent de recenser tous les produits et charges annuels de la ferme afin de dégager les grandeurs économiques essentielles.

Ainsi, plusieurs indices, notamment le produit brut, la valeur ajoutée, le résultat brut d'exploitation et le résultat net d'exploitation ont été calculés pour faire la comparaison entre les différentes fermes. En désignant par Q la quantité des produits récoltés sur une superficie donnée et $\mathrm{PU}$ le prix de vente, le produit brut $(\mathrm{PB})$ est donné par : $\mathrm{PB}=\left(\mathrm{Qx}_{1} \mathrm{x} \mathrm{PUx}_{1}\right)+\left(\mathrm{Qx}_{2} \mathrm{x} \mathrm{PUx}_{2}\right)+\ldots$ $\left(\mathrm{Qx}_{\mathrm{n}} \mathrm{x} \mathrm{PUx}_{\mathrm{n}}\right)$. La valeur ajoutée (VA) correspond à la différence entre le produit brut et la valeur des consommations intermédiaires (CI). Pour cette étude, les consommations intermédiaires représentent seulement les consommations en semences pour les cultures et alevins pour la pisciculture étant donné que celles des fumures (organique et minérale), pesticides, aliments pour bétails et autres sont nulles.

La valeur ajoutée (VA), le résultat brut d'exploitation (RBE) et le résultat net d'exploitation (RNE), sont donnés par les formules de Fabre (1994) cité par Stensens (2002) :

- VA=PB-CI.

- RBE=VA-(rémunération du travail salarié + frais financiers + taxe).

- RNE=RBE-amortissement. 
Le RBE exprime le gain (ou la perte) économique de l'agent une fois acquittées toutes les charges d'exploitation courantes. Le RNE exprime le gain (ou la perte) économique compte tenu des investissements effectués préalablement. Cette analyse de rentabilité prend en compte la valeur ajoutée non incluse la rémunération de la main-d'œuvre familiale. Par ailleurs, en vue de bien établir le compte d'exploitation, l'amortissement de l'équipement et de l'outillage a été pris en compte. Selon Culman (1984), il est nécessaire d'évaluer l'intensité d'utilisation des outils agricoles et la dépréciation en relation avec leur degré d'utilisation. Pour la simplicité des calculs, l'amortissement linéaire a été adopté. Dans tout le corridor de Monkoto, les fermiers (installés à la même période) font deux saisons de cultures par an pour les cultures pluviales annuelles. Par conséquent, une durée effective d'utilisation annuelle des outils de 12 mois sur 12 a été considérée.

Blancard et al. (2013) et Bessan et al. (2018) estiment que l'analyse par des grandeurs économiques est en elle-même insuffisante pour bien mesurer la performance des organisations et qu'elle doit être complétée utilement par d'autres approches multidimensionnelles telles que la méthode d'enveloppement des données «DEA : Data Envelopment Analysis » et la méthode des frontières stochastiques de production. La mesure du degré d'efficacité technique d'une unité de production permet de cerner si cette dernière peut accroître sa production sans pour autant consommer plus de ressources, ou diminuer d'au moins un intrant tout en conservant le même niveau de production (Amara et Robert, 2000). L'efficacité technique mesure la manière dont une firme - cas d'une ferme pilote - choisit les quantités de facteurs de production qui entrent dans le processus de production quand les proportions d'utilisation des facteurs sont données (Farrell, 1957).

Dans cette étude, l'efficacité technique a été évaluée par la méthode DEA. Cette méthode est donc particulièrement adaptée à la mesure de la productivité multi-facteurs relative des fermes pilotes. Le score d'efficacité qu'elle génère en présence de multiples facteurs de production et de multiples produits est défini comme : le rapport entre la somme pondérée des facteurs de production et la somme pondérée des produits (Ale, 2010).

La méthode DEA est un outil d'analyse et d'aide à la décision qui en calculant un score d'efficacité, indique si une ferme dispose d'une marge d'amélioration. Cette approche non-paramétrique ne nécessite aucune hypothèse sur la forme de la fonction de production, de la fonction de coût, ou de profit. Sa particularité est que les poids alloués aux produits et aux facteurs de production ne sont pas choisis par les décideurs (Huguenin, 2013). Le recours à cette technique basée sur la programmation linéaire à l'aide du Solveur MS Excel 2010 (Proudlove, 2000) a permis de mesurer l'efficacité technique des fermes modèles pilotes. 
Le score d'efficacité de chaque ferme est calculé par rapport à une frontière d'efficience. L'efficience évalue l'écart existant entre la situation observée des fermes et leur situation optimale située sur la frontière de production considérée comme une référence à atteindre (Blancard et al., 2013 ; Bessan et al., 2018). Les fermes qui se situent sur la frontière ont un score de 1 (ou 100\%), c'est-à-dire qu'elles sont efficaces. Les fermes qui sont localisées sous la frontière ont un score inférieur à 1 (ou 100\%) - et disposent par conséquent d'une marge d'amélioration de leur performance.

\section{Approches méthodologiques Milieu d'étude}

Les « fermes pilotes » concernées par la présente étude sont situées dans le corridor de Monkoto, dans un rayon de $100 \mathrm{~km}$ maximum au tour du chef-lieu du territoire de Monkoto. Ce dernier est l'un des territoires de la nouvelle province de la Tshuapa issue du démembrement de l'ancienne province de l'Equateur.

D’une superficie générale de $5881,6 \mathrm{Km}^{2}$ et composée essentiellement de la forêt primaire, le Corridor de Monkoto est situé entre les deux blocs du parc national de la Salonga (le Bloc Nord et le Bloc Sud) entre $1^{\circ} 15^{\prime \prime}$ et $2^{\circ} 15^{\prime \prime}$ de latitude sud et $20^{\circ} 30^{\prime \prime}$ et $21^{\circ} 45^{\prime \prime}$ de longitude Est. C'est une Zone à Gestion Communautaire des Ressources Naturelles (ZGCRN) limitée au Nord par le Bloc nord du Parc national de la Salonga ; au Sud par le bloc sud du même parc ; au Nord-Ouest par les secteurs de Bianga et de Nongo et, au Sud-Est par le Territoire de Dekese dans le Kasaï Central et Kole dans le Sankuru.

De manière générale, les principales activités de subsistance et de rente de la population de la ZGCRN Corridor de Monkoto sont : l'agriculture, la chasse, la pêche, l'artisanat et l'élevage.

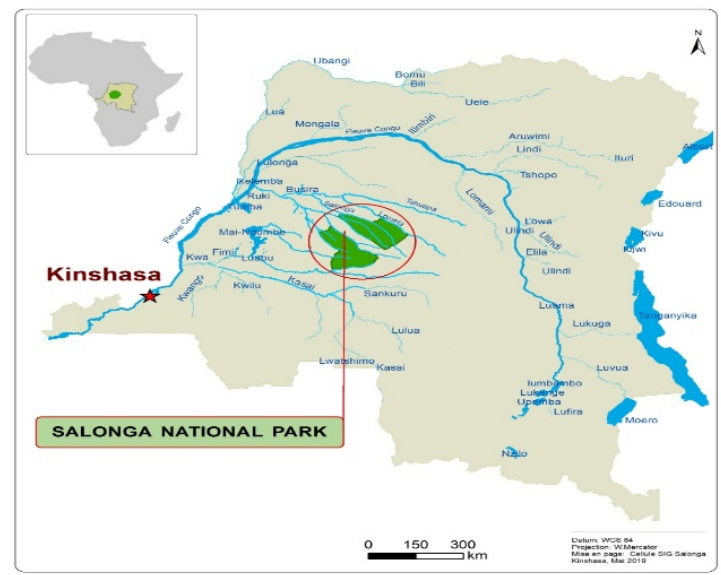

Carte 1 : Présentation et localisation de la zone d'étude (Source : Unité de Gestion du PNS, 2019) 


\section{Collecte, traitement et analyse des données}

Une enquête de terrain, mêlant la récolte de données qualitatives et quantitatives à travers les entretiens et les observations, a été réalisée en septembre 2018 pour recueillir les données nécessaires à la présente étude. Les entretiens individuels approfondis ont été réalisés avec 10 fermiers dont une femme et 9 hommes, localisés dans les différents villages du corridor de Monkoto (Boangi, Bongila, Bonga, Indjolo, Isaka, Iliko, Betambe et Isenga). Individuellement, les fermiers ont répondu à un ensemble de questions en rapport avec les aspects techniques, socio-économiques, financiers et commerciaux des fermes. L'observation (visite à la ferme) a été effectuée pour avoir un aperçu général sur le niveau d'intégration «agriculture-élevagepisciculture » dans la ferme et d'affirmer ou infirmer les dires des fermiers. Cette approche (entretien individuel et observation) a permis de collecter les données par la théorie de confrontation directe d'informations (Allou et Fofana, 2018) en vue de choisir les plus pertinentes pour cette étude. A côté de ces 10 fermes pilotes mises en place au premier trimestre de l'année 2017, les 10 autres fermes promues à la réplication sur base du même modèle ont été visitées mais ces dernières n'ont pas montré d'intérêt pour cette étude étant donné qu'aucune activité n’avait été mise en place au moment de la collecte de données.

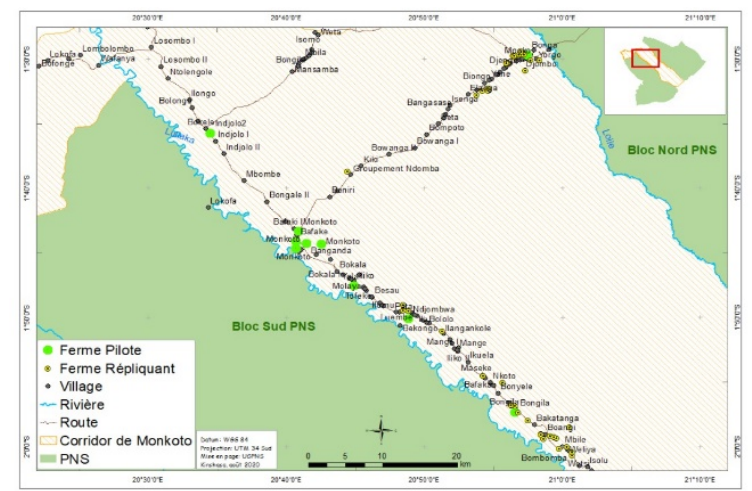

Carte : Localisation des fermes pilotes dans le corridor de Monkoto (Source : Unité de Gestion du PNS, 2020)

La grande diversité des types d'exploitations agricoles est une difficulté pour évaluer les performances économiques des pratiques agricoles locales (Vall et al., 2011; Gafsi et Favreau, 2014; Pallière et Rangé, 2017). Cependant, dans le cadre de cette étude, les fermes modèles pilotes sont aussi semblables que possible, ce qui a facilité l'analyse et la comparaison des résultats (Blancard et al., 2013). En effet, situées dans la même région agricole (corridor de Monkoto), ces exploitations sont toutes placées dans des conditions identiques de potentialités naturelles (les conditions climatiques, la 
nature et la fertilité des sols, l’hétérogénéité des terres, etc.) et socioéconomiques (l'accès aux facteurs de production, les débouchés commerciaux, le niveau des prix, etc.).

Les informations recueillies à travers les enquêtes sur le terrain ont subi un dépouillement manuel et informatique. Quant aux données qualitatives, une analyse du contenu a été effectuée. Elle a permis après découpage et retranscription, d'organiser les informations selon les thématiques discutées avec les acteurs interrogés. Le pack office word a été le principal outil informatique utilisé pour l'analyse et le rapportage. Le logiciel SPSS a servi à faire les analyses statistiques liées aux données quantitatives alors que le pack Excel a été utilisé pour l'analyse DEA à l'aide de son solveur et l'élaboration de tableaux et graphiques.

\section{Résultats et discussion}

\section{Systèmes de production des fermiers pilotes}

\section{Production agricole}

La production agricole est très diversifiée dans les fermes (polyculture) et concerne les cultures vivrières, fruitières et horticoles. Les cultures vivrières exploitées dans ces fermes sont principalement le manioc, le maïs, le riz et dans des rares cas le niébé, la courge et l'arachide (tableau 1). Le manioc est la culture dominante et relativement importante (90\% des fermes). Il constitue l'aliment de base dans la zone avec des chaines de valeur telles que la chikwangue et la farine de manioc, utile dans la préparation du fufu et d'autre part, comme source de revenu par la vente de, « lotoko », l'alcool indigène. C'est plus pour ce dernier usage que le maïs est cultivé, la quasi-totalité de la production étant utilisée comme matière première dans la fabrication de l'alcool traditionnel, importante source de revenu.

$\mathrm{Au}$ sein des fermes modèles, le rendement moyen du manioc est de 16 tonnes/ha. Ce rendement est largement supérieur à celui de 7,5 tonnes/ha obtenu par les petits exploitants dans la zone (ITAPEL, 2017) et celui de plus de 7,9 t/ha en moyenne soit 25 sacs des cosettes en Afrique. Il est égal à celui du Niger (meilleur rendement en Afrique) et largement inférieur à celui de l'Inde (meilleur rendement au monde) qui sont respectivement évalués à 16,3 t/ha et 29 t/ha en moyenne (Soule et al., 2013).

Le maïs est exploité par 3 fermes sur 10. Si la superficie moyenne n’est pas différente de celle pratiquée par les paysans, le rendement moyen de 0,91 tonnes/ha est supérieur à celui obtenu en milieu paysan soit 0,61 tonnes/ha (ITAPEL, 2017). Ce rendement est supérieur à celui observé dans la province du Kongo central soit 0,778 tonnes/ha (Mpanzu et al., 2011). Il reste toutefois largement inférieur à celui observé dans la plaine de la Ruzizi (5 tonnes/ha) au Sud Kivu (Fuhara, 2017) mais également en Afrique de 
l'Ouest, notamment le Sénégal (1,5 tonnes/ha), le Mali (2,8 tonnes), la Côte d'Ivoire (2 tonnes) et le Ghana (1,9 tonnes) (Gnimadi, 2008).

Le riz présente une véritable source de revenu pour les fermiers (photo1). La culture du riz est pratiquée dans 7 fermes sur 10 avec notamment 5 fermes pour le riz irrigué et 2 fermes pour le riz pluvial, mais avec des pépinières de riz irrigué en attente du repiquage. Les superficies emblavées sont encore trop faibles par rapport au potentiel rizicole de la zone. Ceci est dû à des périmètres rizicoles caractérisés par des faibles aménagements hydro agricoles. Avec 0,53 ha en moyenne contre 0,23 ha en milieu paysan et 0,97 ha dans la plaine de la Ruzizi au Sud Kivu et 70 ares au Mali, le rendement moyen y est de 1,6 tonnes/ha contre 1,42 tonnes en milieu paysan. Ce rendement est supérieur à celui observé en RDC, soit une moyenne de 0,685 tonnes/ha au niveau national en 2015 ; mais il reste largement inférieur à celui observé dans les pays du Sahel, notamment le Mali qui dégage un rendement de 5 à 6 tonnes/ha, avec des pointes de 8 t/ha en contre saison (ITAPEL, 2017 ; Fuhara, 2017).

Les fermiers déclarent vendre plus de $80 \%$ de leur production de riz alors que le taux de commercialisation dépasse les $90 \%$ pour la courge, le niébé et l'arachide.

Tableau 2. Performances moyennes des principales cultures vivrières pratiquées par les fermiers

\begin{tabular}{|l|l|l|l|l|l|l|l|l|l|l|}
\hline & \multicolumn{9}{|l|}{ Superficie exploitée $(\mathrm{Ha})$} & \multicolumn{6}{l|}{ Production (Tonne) } & \multicolumn{2}{l|}{$\begin{array}{l}\text { Rendement } \\
\text { (Tonne/Ha) }\end{array}$} \\
\hline Ferme & $\begin{array}{l}\text { Manio } \\
\text { c }\end{array}$ & Maïs & Riz & $\begin{array}{l}\text { Manio } \\
\text { C }\end{array}$ & Maïs & Riz & $\begin{array}{l}\text { Manio } \\
\text { C }\end{array}$ & Maïs & Riz \\
\hline Bafake & 0,7 & & 0,64 & 10 & & 1,1 & 14,3 & & 1,7 \\
\hline Bonga & 3,1 & 0,38 & 0,8 & 42 & 0,25 & 1,2 & 13,5 & 0,7 & 1,5 \\
\hline Iliko & 1,3 & & & 20 & & & 15,4 & & \\
\hline Betamba 1er & 2,2 & & 0,232 & 41 & & 0,4 & 18,6 & & 1,7 \\
\hline Salongo 1 & & & & & & & & & \\
\hline Isaka & 0,5 & & & 8 & & & 16,0 & & \\
\hline Salongo 2 & 0,7 & & 0,61 & 10 & & 1,0 & 14,3 & & 1,6 \\
\hline Indjolo 2 & 2 & 1,1 & 0,31 & 32 & 1,3 & 0,5 & 16,0 & 1,2 & 1,6 \\
\hline Air Congo & 1,9 & & 0,5 & 30 & & 0,8 & 15,8 & & 1,6 \\
\hline Bongila & 3,6 & 2 & 0,6 & 70 & 1,8 & 0,9 & 19,4 & 0,9 & 1,5 \\
\hline Moyenne & 1,8 & 1,2 & 0,53 & 29,2 & 1,1 & 0,8 & 15,9 & 0,9 & 1,6 \\
\hline
\end{tabular}

Source : Auteurs, enquête fermes modèles pilotes, septembre 2018

Le développement des cultures maraîchères est fortement lié à la pluviométrie (l'eau des étangs pouvant être utilisée en cas de besoin) mais aussi à la disponibilité des semences. Semés à la volée et en intercalaire, la superficie occupée par les légumes reste difficile à évaluer. Les cultures pratiquées sont nombreuses (patate douce, oseille, amarante, aubergine, 
tomate, etc.) dont la production est en grande partie autoconsommée. A ce jour, ces cultures ne présentent pas un intérêt économique pour les fermiers dans la zone, c’est ainsi qu'ils y accordent peu d'attention.

Les cultures fruitières sont exploitées dans 8 fermes sur 10. On y trouve en ordre d'importance l'avocatier et l'ananas dans 7 fermes sur 10, suivis de l'oranger, du safoutier, du palmier à huile et du bananier dans 5 fermes sur 10. Dans des rares cas, les fermiers s'adonnent aussi à la culture du cocotier et de la canne à sucre. Les mobiles d'exploitation de deux premiers groupes de cultures sont essentiellement marchands.

Il y a dans les fermes pilotes, quelques rares associations agrisylvicoles du type verger/céréales, racines et parfois légumineuses. Du point de vue agro écologique, s’il a été impossible de dégager la capacité de séquestration des telles associations, on peut noter quelques impacts positifs observables au niveau des fermes pilotes. Sur le plan de la biodiversité notamment, avec une dizaine d'espèces fruitières présentes dans la plupart des fermes, l'agroforesterie occasionne une biodiversité et une variabilité génétique élevées en plus de contribuer significativement à l'amélioration continue de la couverture du sol et du microclimat au sein des fermes. Partant de la régulation thermique, le dessous des arbres sert d'endroit de repos pendant les temps chauds tant pour les hommes que pour les animaux.

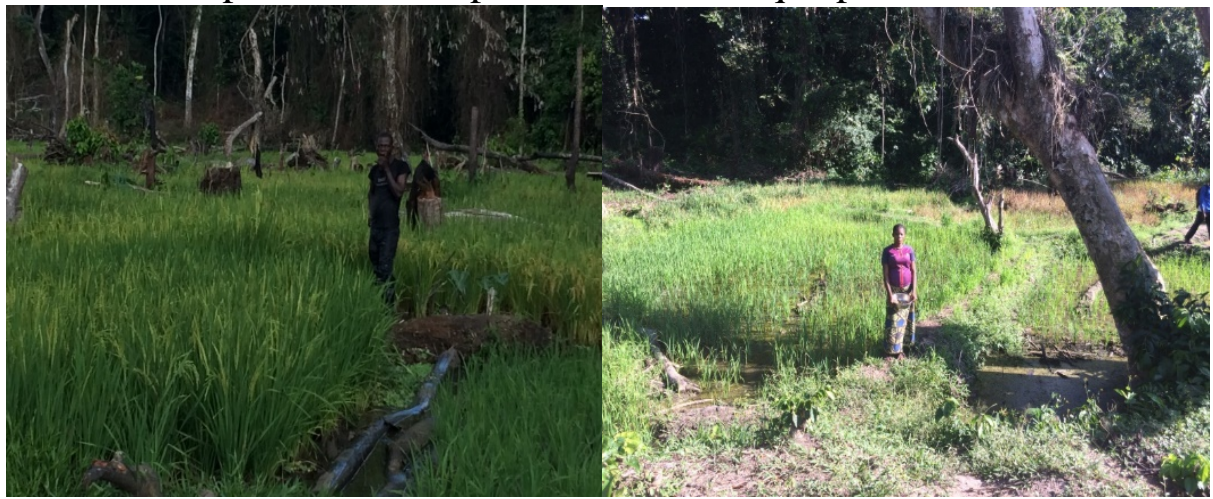

Photo 1 : Valorisation des bas-fonds par la riziculture

(Source : Enquête fermes modèles, septembre 2018)

\section{Production animale et pisciculture}

La pisciculture parait l'activité phare des fermiers pilotes à côté du riz irrigué dans la mesure où bon nombre d'entre eux soit 7 fermiers sur 10, y résument la compréhension et la substance même de la ferme modèle (photo 2).

Cette activité est pratiquée par l'ensemble de fermiers mais à des degrés bien différents quant à l'aménagement et l'entretien des étangs En moyenne, chaque fermier a trois étangs de 10 ares de superficie (tableau 2). La plus grande superficie piscicole a été trouvée à la ferme Bafake avec un 
peu plus de 20 ares alors que la ferme Betambe $1^{\text {er }}$ dispose de la plus petite surface piscicole (environ 1,5 are). Les fermiers y exploitent les espèces locales des poissons telles que le tilapia et le clarias.

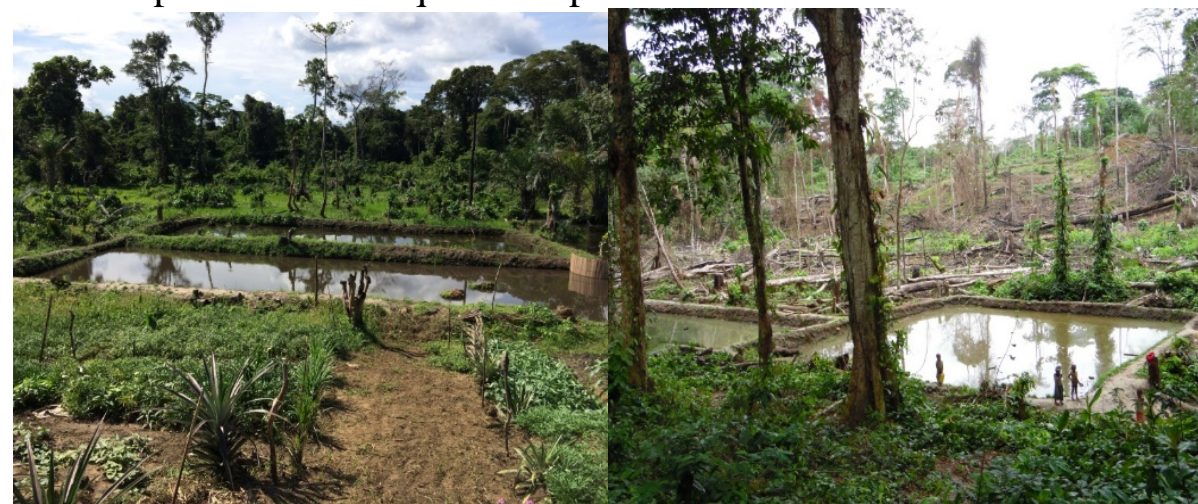

Photo 2 : Valorisation des bas-fonds par les étangs piscicoles (ferme Bongila)

(Source : Enquête fermes modèles, septembre 2018)

L'élevage intégré à l'exploitation agricole commence à évoluer lentement dans les fermes pilotes (tableau 2). Sur les 9 fermiers qui font l'élevage, seulement 3 (Bafake, Salongo 2 et Air Congo) font l'élevage extensif des animaux de race locale dans la ferme contre 6 autres (Bonga, Iliko, Betambe $1^{\mathrm{er}}$, Isaka, Indjolo et Bongila) qui ont déclaré faire l'élevage à leur domicile. Dans cette dernière catégorie, la ferme Bongila a le plus cheptel, soit 35 poules, 10 chèvres, 14 moutons et 12 porcs. Etant donné l'inexistence ou la faiblesse d'infrastructures adéquates, l'absence de bétail dans la plupart de fermes prive l'exploitation des apports organiques et limite ainsi l'intégration agriculture et élevage pourtant caractéristique majeure du modèle agricole (Dugué et al, 2004). Ceci prive aussi les fermiers d'un revenu monétaire, étant donné que le troupeau et sa multiplication constituent «le mécanisme d'accumulation du capital de l'exploitation » (Minani, 2014). Le taux annuel de vente des bétails est en moyenne de $38 \%$. Véritable filet de sécurité pour les ménages pauvres, en particulier ceux du territoire de Monkoto, l'élevage constitue également une forme d'épargne permettant de faire face à différents problèmes (ils peuvent être vendus, consommés à des occasions particulières ou utilisés comme moyen de payement). Cet élevage est aussi une source de protéines pour les ménages de fermiers, véritable alternative à la viande de chasse dans le contexte de la zone d'étude.

Tableau 3. Nombre d'étangs et d'animaux dans les fermes modèles

\begin{tabular}{|c|c|c|c|c|c|c|c|}
\hline & \multicolumn{3}{|c|}{ Pisciculture } & \multicolumn{4}{c|}{ Elevage } \\
\hline Ferme & $\begin{array}{c}\text { Nombre } \\
\text { d'étang }\end{array}$ & $\begin{array}{c}\text { Superficie } \\
\text { totale } \\
\text { (ares) }\end{array}$ & $\begin{array}{c}\text { Superficie } \\
\text { moyenne } \\
\text { (ares) }\end{array}$ & $\begin{array}{c}\text { Nombre de } \\
\text { poules }\end{array}$ & $\begin{array}{c}\text { Nombre de } \\
\text { chèvres }\end{array}$ & $\begin{array}{c}\text { Nombre de } \\
\text { moutons }\end{array}$ & $\begin{array}{c}\text { Nombre } \\
\text { de porcs }\end{array}$ \\
\hline Bafake & 3 & 20,4 & 6,8 & 30 & 8 & 2 & 1 \\
\hline
\end{tabular}




\begin{tabular}{|c|c|c|c|c|c|c|c|}
\hline Bonga & 3 & 17,8 & 5,9 & & & & \\
\hline Iliko & 2 & 3,0 & 1,5 & & & & \\
\hline Betamba 1er & 2 & 1,5 & 0,7 & & & & \\
\hline Salongo 1 & 4 & 6,8 & 1,7 & & & & \\
\hline Isaka & 3 & 7,5 & 2,5 & & & & \\
\hline Salongo 2 & 3 & 7,0 & 2,3 & 25 & 0 & 0 & 0 \\
\hline Indjolo 2 & 3 & 9,0 & 3,0 & & & & \\
\hline Air Congo & 5 & 12,36 & 2,5 & 20 & 4 & & \\
\hline Bongila & 4 & 11,0 & 2,8 & & & & 1 \\
\hline Moyenne & 3,2 & 9,6 & 3,0 & 25 & 4 & 1 & 1 \\
\hline
\end{tabular}

Source : Enquête fermes modèles, septembre 2018

La taille moyenne (3 ares) des étangs piscicoles démontre que la pisciculture telle que pratiquée par les fermes modèles est encore loin des standards d'une pisciculture commerciale dont la taille moyenne des étangs varie entre 4 et 10 ares pour la pisciculture commerciale à petite échelle et 10 à 50 ares pour la pisciculture commerciale à grande échelle (ACF, 2008). Comme dans la plaine de la Ruzizi, à côté de faibles superficies des étangs, les pratiques piscicoles dans la zone sont caractérisées notamment par une utilisation limitée de l'eau, pas d'utilisation d'aliments et engrais, une récolte faible et orientée pour le marché local (IRC, 2018).

\section{Utilisation et valorisation des facteurs de production par les fermiers} Terre

Toutes les fermes évoluent en faire valoir direct. Les fermiers sont propriétaires des terres qu'ils exploitent. Cette propriété est principalement acquise par l'abattage d'une forêt primaire sur l'accord verbal du chef de la localité, l'appartenance des terres au clan faisant d'eux des ayants droit coutumier. Seulement 2 fermiers sur 10 ont acquis la terre par achat mais comme les propriétaires, ils n'en détiennent aucun document officiel.

Les fermes modèles pilotes exploitent partiellement leurs terres (au plus 30\% des terres disponibles). En moyenne la superficie disponible est de 15 ha $\pm 10,8$ et celle exploitée, de seulement 3,5 ha $\pm 2,4$. La superficie moyenne exploitée par les fermes modèles pilotes reste largement supérieure à celle des exploitations agricoles familiales en milieu paysan en RDC qui est de 0,35 ha (Chausse et al., 2012 ; RDC, 2013). En RDC, les estimations des superficies moyennes exploitées par les ménages varient d'une province à l'autre, comme le montrent les résultats forts variés selon les sources : 1,6 ha en moyenne dans la province du Kasaï (Miteu et al., 2019) ; moins d'un ha dans la province du Kongo Central (Mpanzu et al., 2011). Comme le démontre la dispersion autour de la moyenne, il existe des disparités tant dans la 
disponibilité des terres que dans l'exploitation de ces dernières en ce sens que $20 \%$ des fermiers pilotes emblavent moins d' 1 ha, $60 \%$ exploitent entre 1 et 5 ha et $20 \%$ entre 5 et 7 ha (figure 1 ).

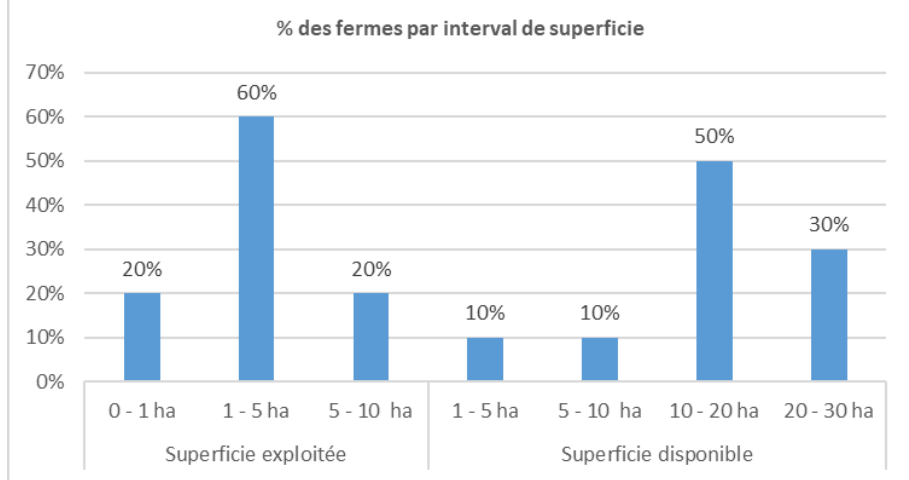

Figure 1.Superficie disponible et exploitée

L'écart entre la superficie des terres disponibles et celles exploitées est plus lié au manque de moyens financiers et techniques qu'à des considérations agro écologiques. En plus de ces terres exploitées, dans leur ensemble les fermiers et/ou les membres de leurs familles exploitent d'autres terres en dehors de la ferme. Ils y font des cultures vivrières et/ou horticoles qui contribuent très largement à leur alimentation.

\section{Capital}

Alors que la disponibilité et l'usage des équipements agricoles modèles seraient ainsi associés à une performance tout à fait particulière des exploitations agricoles (Hanafi et al., 2015); les fermes modèles pilotes de la zone d'étude sont malheureusement caractérisées par une faible intensité capitalistique. Elles ne détiennent que 2 houes, 2 machettes, 3 bèches/pelles, 1 hache en moyenne pour toutes leurs opérations culturales. En outre, certains fermiers utilisent des matériels tels que l'arrosoir, les bassines, les paniers, et autres. Partant du prix d'achat, du nombre d'outils et équipements utilisés et de leur durée de vie, la méthode d'amortissement linéaire a permis de dégager les dotations d'amortissement (tableau 3).

Tableau 4.Coût moyen annuel de l'amortissement des équipements et outillage de production

\begin{tabular}{|c|c|c|c|c|}
\hline Ferme & $\begin{array}{c}\text { Superficie } \\
\text { exploitée (ha) }\end{array}$ & $\begin{array}{c}\text { Valeur totale } \\
\text { actuelle du } \\
\text { Matériel (CDF) }\end{array}$ & $\begin{array}{c}\text { Annuité d'amortissement } \\
(\mathrm{CDF})\end{array}$ & $\begin{array}{c}\text { Annuité } \\
\text { d'amortissement } \\
\text { /Ha }\end{array}$ \\
\hline Bafake & 2 & 99500 & 128000 & 64000,0 \\
\hline Bonga & 7 & 54500 & 68000 & 9714,3 \\
\hline Iliko & 3 & 94500 & 115500 & 38500,0 \\
\hline $\begin{array}{c}\text { Betamba } \\
\text { 1er }\end{array}$ & 5 & 77000 & 116000 & 23200,0 \\
\hline
\end{tabular}




\begin{tabular}{|c|c|c|c|c|}
\hline $\begin{array}{c}\text { Salongo } \\
1\end{array}$ & 0,07 & 89500 & 130000 & 1911764,7 \\
\hline Isaka & 1 & 107000 & 146000 & 146000,0 \\
\hline $\begin{array}{c}\text { Salongo } \\
2\end{array}$ & 1,5 & 68000 & 92000 & 61333,3 \\
\hline Indjolo 2 & 5 & 117000 & 156000 & 31200,0 \\
\hline $\begin{array}{c}\text { Air } \\
\text { Congo }\end{array}$ & 3 & 190500 & 219000 & 73000,0 \\
\hline Bongila & 7 & 377500 & 508000 & 72571,4 \\
\hline Moyenne & 3,5 & 173600 & 167850 & 48556,5 \\
\hline
\end{tabular}

Source : Auteurs, enquête fermes modèles pilotes, septembre 2018

Etant donné que ces fermes exercent dans une zone forestière qui provoque une détérioration rapide des équipements et outils et la perte totale de leur valeur sur une seule saison, seul l'arrosoir a été amorti en considérant sa durée de vie économique estimée à 2 ans. Certains matériels sont renouvelés au moins deux fois dans la même année, c'est le cas de la machette, la lime et le panier. Ainsi, chaque ferme devrait constituer les dotations d'amortissement de $48.600 \mathrm{CDF}$ en moyenne pour être à mesure de renouveler l'équipement de production après usure.

Le gros de semences utilisées par les fermiers provient des précédentes récoltes. Celles-ci sont complétées par les semences achetées sur le marché local ou chez d'autres petits fermiers de la zone et donc, la disponibilité des semences détermine non seulement les spéculations à exploiter mais aussi les superficies à emblaver par les fermes. Tshomba et al. (2019) font remarquer que la stagnation des superficies agricoles emblavées en RDC entre 2000 et 2013 serait due à l'accès difficile aux intrants agricoles, conduisant dans $95 \%$ des cas, les producteurs à réduire jusqu'à la moitié la superficie exploitée. L'accès aux intrants reste donc un enjeu dans l'aboutissement et la réplication du modèle agricole des fermes pilotes.

Par rapport aux cultures vivrières, le coût moyen des semences (ou boutures) par ha est de 80.000 CDF pour le riz, 60.000 CDF pour le manioc, 20.000 CDF pour le maïs et la courge, 25.000 CDF pour le niébé et l'arachide et $33.000 \mathrm{CDF}$ pour la patate douce. En ce qui concerne les cultures fruitières, les besoins des plantules par ha se chiffrent à 200.000 CDF pour l'oranger, l'avocatier, le safoutier, le cocotier et le palmier à huile, 400.000 CDF pour le bananier et 250.000 CDF pour l'ananas. Les informations sur les écartements ont permis de déterminer la densité et par voie de conséquences, le nombre des plantules nécessaires pour couvrir 1 ha. Dans la zone, une plantule coûte généralement 1.000 CDF pour différentes cultures sauf l'ananas dont le rejet coûte $100 \mathrm{CDF}$.

En ce qui concerne la pisciculture, les alevins sont achetés soit chez d'autres pisciculteurs soit chez les pêcheurs au coût estimé de 1.000 CDF par 
are alors qu'un poussin couterait 1.600 CDF, contre 16.000 CDF pour un chevreau et un agneau, et 32.000 CDF pour un porcelet dans le cadre de l’élevage.

Si dans certaines fermes, on a noté la présence de Mucuna comme engrais organique, il a été difficile d’évaluer le coût étant donné que la valeur de cette plante aux fins évoquées n’est pas du tout exploitée actuellement. La totalité des fermes n'utilisent ni la fumure minérale ni les produits phytosanitaires. Ce poste des charges est donc nul et par voie de conséquences, dans l'analyse des performances, les consommations intermédiaires sont composées des semences et alevins.

\section{Travail}

La main d’œuvre représente la force de travail utilisée pour l'exécution des opérations culturales. Dans l'absence totale de la mécanisation et de la traction animale, la main-d'œuvre familiale permanente et la maind'œuvre salariée temporaire constituent les différentes formes de travail utilisées par les fermiers. La main-d’œuvre salariée est utilisée par tous les fermiers dans toutes les opérations culturales depuis la préparation du sol jusqu’à la récolte voire les opérations de valorisation du produit de récolte.

En ouverture de forêt primaire, le coût global de la main d'œuvre selon les opérations effectuées pour un ha de culture de manioc se présente comme suit :

Tableau 5.Quantité et coût de la main d'ouvre pour 1 ha de manioc

\begin{tabular}{|c|c|c|c|}
\hline Opérations & $\begin{array}{c}\text { Nombre d'H- } \\
\boldsymbol{J}\end{array}$ & $\begin{array}{c}\text { Coût unitaire } \\
\text { (CDF) }\end{array}$ & Coût total (CDF) \\
\hline Délimitation terrain & 4 & 2500 & 10.000 \\
\hline Coupe sous-bois & 50 & 2500 & 125.000 \\
\hline Abatage & 60 & 2500 & 150.000 \\
\hline Débitage & 40 & 2500 & 100.000 \\
\hline Incinération & 2 & 2500 & 5.000 \\
\hline Ramassage & 60 & 2500 & 150.000 \\
\hline Labour & 50 & 2500 & 125.000 \\
\hline Bouturage & 40 & 2500 & 100.000 \\
\hline Regarnissage des vides & 20 & 2500 & 50.000 \\
\hline Sarclage & 40 & 2500 & 100.000 \\
\hline Récolte (y compris l'épluchage) & 50 & 2500 & 125.000 \\
\hline Séchage ou égrainage & 20 & 2500 & 50.000 \\
\hline Mise en sac & 4 & 2500 & 10.000 \\
\hline Total & & & $\mathbf{1 . 1 0 0 . 0 0 0}$ \\
\hline
\end{tabular}

Source : Auteurs, enquête fermes modèles pilotes, septembre 2018

La quantité et le coût de la main d'œuvre varient pour certaines activités selon la culture et la zone. La pénibilité de la tâche et la disponibilité de la main d’œuvre dans la zone en sont des déterminants essentiels. 
Dans toutes les fermes, les opérations de semis, d'entretien et de récolte sont plus exigeantes pour le riz que pour les autres cultures, notamment le manioc et le maïs avec respectivement $50 \mathrm{HJ}$ contre 40 et $20 \mathrm{HJ}$ pour le semis, $50 \mathrm{HJ}$ contre 40 et $20 \mathrm{HJ}$ pour le sarclage, $100 \mathrm{HJ}$ contre 50 pour la récolte et $10 \mathrm{HJ}$ contre 10 et $4 \mathrm{HJ}$ pour la mise en sac (tableau 4).

Quant aux cultures vivrières, les opérations culturales vont de la délimitation du terrain à l'incinération. L’aménagement des étangs piscicoles est aussi précédé des mêmes opérations étant donné la nature du terrain (tableau 5).

Tableau 6.Quantité et coût de la main d'œuvre pour la construction des étangs piscicoles

\begin{tabular}{|c|c|c|c|}
\hline Opérations & Nombre d'H-J & Coût unitaire (CDF) & Coût total (CDF) \\
\hline Délimitation terrain & 4 & 2500 & 10.000 \\
\hline Coupe sous-bois & 50 & 2500 & 125.000 \\
\hline Abatage & 60 & 2500 & 150.000 \\
\hline Débitage & 40 & 2500 & 100.000 \\
\hline Incinération & 2 & 2500 & 5.000 \\
\hline Plan d'aménagement & 120 & 2500 & 300.000 \\
\hline Construction des digues & 60 & 2500 & 150.000 \\
\hline Total & & & $\mathbf{8 4 0 . 0 0 0}$ \\
\hline
\end{tabular}

Source : Auteurs, enquête fermes modèles pilotes, septembre 2018

La main-d'œuvre salariée représente pour l'ensemble 69,2\% de la main-d'œuvre totale utilisée (tableau 6). Le travail salarial a alors un poids très important dans la production agricole dans les fermes pilotes. Lors des périodes de pointe des travaux agricoles, les fermiers ont alors besoin d'une liquidité importante pour faire face au paiement des ouvriers agricoles. C'est ainsi que, parmi les contraintes à la production, l'insuffisance des ressources financières est plus évoquée (8 fermes sur 10). Ce qui limite aussi en grande partie l'extension de la superficie des fermes pilotes.

Tableau 7.Proportion et coût de type de la main d'œuvre par ferme

\begin{tabular}{|c|c|c|c|c|c|}
\hline Ferme & $\begin{array}{c}\text { MO } \\
\text { familiale (\%) }\end{array}$ & $\begin{array}{c}\text { Coût de la MO } \\
\text { familiale } \\
\text { (CDF) }\end{array}$ & $\begin{array}{c}\text { MO salariée } \\
\text { (\%) }\end{array}$ & $\begin{array}{c}\text { Coût de la MO } \\
\text { salariée (CDF) }\end{array}$ & $\begin{array}{c}\text { Coût Total } \\
\text { MO (CDF) }\end{array}$ \\
\hline Befake & 34,5 & 740543,2 & 65,5 & 1405958,8 & 2146502,0 \\
\hline Bonga & 17,5 & 279082,6 & 82,5 & 1315675 & 1594757,6 \\
\hline Iliko & 30,0 & 635656,8 & 70,0 & 1483199,2 & 2118856,0 \\
\hline Betamba 1er & 29,6 & 302630,4 & 70,4 & 719769,6 & 1022400,0 \\
\hline Salongo 1 & 16,7 & 211088,0 & 83,3 & 1052912,0 & 1264000,0 \\
\hline Isaka & 25,1 & 392832,1 & 74,9 & 1175359,9 & 1568192,0 \\
\hline Salongo 2 & 18,8 & 300765,4 & 81,3 & 1303316,6 & 1604082,0 \\
\hline Indjolo 2 & 34,1 & 1087720,4 & 66,0 & 2106759,6 & 3194480,0 \\
\hline Air Congo & 46,8 & 1348785,4 & 53,2 & 1533234,6 & 2882020,0 \\
\hline Bongila & 55,4 & 2305543,4 & 44,7 & 1859846,6 & 4165390,0 \\
\hline Moyenne & 30,8 & 760464,8 & 69,2 & 1395603,2 & 2156068,0 \\
\hline
\end{tabular}

Source : Auteurs, enquête fermes modèles pilotes, septembre 2018 
Trois fermes (Bonga, Salongo 1 et Salongo 2) ont un taux de main d'œuvre familiale inférieur à 20\%. Les responsables de deux premières fermes situées au centre de Monkoto dans le quartier Salongo ont l'enseignement supérieur pour activité principale. Ils passent moins de temps à la ferme alors que le responsable de la ferme de Bonga a moins d'actifs agricoles dans son ménage. La plupart de ses enfants sont dans d'autres activités dans la zone.

\section{Analyse de la performance des fermes modèles}

L'analyse de la performance des fermes se base sur la rentabilité économique, c'est-à-dire du point de vue de toute la ferme et non du point de vue du fonds propres qui ferait appel à l'analyse de la rentabilité financière. A côté de celle-ci, l'analyse de l'efficacité technique, permet de savoir dans quelle mesure les technologies utilisées par les fermiers permettent d'optimiser leurs rendements.

\section{Efficacité technique des fermes modèles}

Toute activité de production met enjeu des intrants, qui sont les ressources utilisées, et des productions. L'évaluation des performances repose sur l'analyse de la valorisation des intrants par des productions. La superficie, la main d'œuvre, la quantité de semences et d'alevins d'une part et la quantité produite de riz paddy, poissons, cossettes de manioc et maïs d'autre part ont permis d'analyser l'efficacité technique et d'évaluer le niveau maximal de production possible pour un niveau donne' d'intrant (tableau 7).

Tableau 8.Variables (in put et ou put) retenues pour l'analyse de l'efficience

\begin{tabular}{|c|c|}
\hline In put (intrants) & Out put (Production estimée en $\mathbf{K g}$ ) \\
\hline Superficie emblavée (ares) & Riz paddy \\
\hline Main d'œuvre $(\mathrm{H}-\mathrm{J})$ & Poisson \\
\hline Semences $(\mathrm{Kg})$ & cossettes \\
\hline Alevins $(\mathrm{kg})$ & Maïs \\
\hline
\end{tabular}

Sous l'hypothèse que les fermes évoluent dans une situation des rendements d'échelle variable étant donné qu'elles n'opèrent pas à leur niveau optimal, les activités des fermes modèles pilotes ont donné les scores d'efficience suivants après analyse :

Tableau 9.Score d'efficience (\%) des fermes modèles pilotes

\begin{tabular}{|c|c|}
\hline Ferme & Score d'efficience (\%) \\
\hline Bongila & 0,082 \\
\hline Bonga & 0,035 \\
\hline Iliko & 0,108 \\
\hline Betamba $1^{\text {er }}$ & 0,021 \\
\hline Salongo 1 & 0,018 \\
\hline
\end{tabular}




\begin{tabular}{|c|c|}
\hline Isaka & 0,032 \\
\hline Salongo 2 & 0,021 \\
\hline Indjolo & 0,235 \\
\hline Air Congo & 0,316 \\
\hline Bongila & 0,398 \\
\hline Moyenne & $\mathbf{0 , 1 2 6 6}$ \\
\hline
\end{tabular}

Source : Auteurs, enquête fermes modèles pilotes, septembre 2018

Avec une moyenne de $12,7 \%$, le score d'efficience relative est faible pour toutes les fermes. Il varie de 1,8\% pour la ferme de Salongo 1 à 39,8\% pour la ferme de Bongila (tableau 8). C’est-à-dire qu'avec une faible intensité capitalistique et des pratiques agricoles extensives, ces fermes n’opèrent pas à leur niveau optimal. Autrement dit, elles ont une taille et un volume d'activité qui ne leur permettent pas de minimiser leur consommation moyenne de ressources, notamment la terre (Al Awamia, 2014). On peut parler du gaspillage des ressources.

Alors que Djimasra (2012) fait remarquer que les exploitations agricoles des pays en développement ont des scores d'efficience technique très bas que celles des pays développés, au Maroc par exemple, entre 1986-2012, le score de l'efficacité technique dans le secteur agricole a été de $90 \%$. En plus, faisant référence aux travaux de Nyemeck et al. (2006), l'auteur présente un niveau d'efficacité d'échelle moyen de $76,6 \%$ pour 81 exploitations de café en étudiant les facteurs qui affectent la filière café dans la région du centre ouest en Côte d’Ivoire. La performance étant liée en grande partie au progrès technique (Azeroual et Doumi, 2016), cette différence de performance est le signe de grandes divergences entre les exploitations et révèle un fort potentiel de rattrapage de l'agriculture africaine.

Dans leur modèle agricole, les intrants ne sont pas bien utilisés et les pratiques agricoles ne sont pas encore maitrisées pour atteindre la production possible étant donné le fort potentiel agro écologique de la zone. Les fermes qui ont le score d'efficacité relative élevé ont existé avant les autres et adopté des techniques modèles de cultures. Et donc, ces pratiques observées devront être mémorisées ou documentées pour servir de référence afin d'améliorer l'efficacité et faciliter l'apprentissage par les autres petits fermiers de la zone.

L’orientation retenue étant l'orientation produit, le score d'efficacité de $39,8 \%$ de la ferme de Bongila signifie que la ferme pourrait augmenter sa production globale (produit) de 60,2\% (100 - 39,8) avec le même volume d'in put (terre, semences, plantules, alevins, main d’œuvre, outils aratoires). 


\section{Rentabilité économique des fermes modèles pilotes}

L'analyse financière a été faite pour dégager les performances économiques des fermes pilotes, le RNE (Revenu Net de l'Exploitation) représente la rentabilité économique des fermes pilotes (tableau 9).

Tableau 10. Compte de résultat prévisionnel annuel des fermes modèles pilotes (CDF)

\begin{tabular}{|c|c|c|c|c|c|c|c|}
\hline & $\boldsymbol{P B}$ & $\boldsymbol{C I}$ & $\boldsymbol{V A}$ & $\boldsymbol{M O S}$ & $\boldsymbol{R B E}$ & Dot amort & $\boldsymbol{R N E}$ \\
\hline Bafake & $1.918 .000,0$ & $263.966,7$ & $1.654 .033,3$ & $1.405 .958,8$ & $248.074,5$ & $128.000,0$ & $120.074,5$ \\
\hline Bonga & $1.196 .000,0$ & $240.440,0$ & $955.560,0$ & $1.770 .864,2$ & $-815.304,2$ & $68.000,0$ & $-883.304,2$ \\
\hline Iliko & $1.750 .325,0$ & $223.150,0$ & $1.527 .175,0$ & $1.502 .551,4$ & $24.623,6$ & $115.500,0$ & $-91.201,4$ \\
\hline Betambe 1 & $900.000,0$ & $44.700,0$ & $855.300,0$ & $1.511 .137,4$ & $-655.837,4$ & $116.000,0$ & $-771.837,4$ \\
\hline Salongo 1 & $1.000 .000,0$ & $68.000,0$ & $932.000,0$ & $1.788 .036,2$ & $-856.036,2$ & $130.000,0$ & $-986.036,2$ \\
\hline Isaka & $1.430 .000,0$ & $140.366,7$ & $1.289 .633,3$ & $1.608 .803,2$ & $-319.169,9$ & $146.000,0$ & $-465.169,9$ \\
\hline Salongo 2 & $1.414 .000,0$ & $165.800,0$ & $1.248 .200,0$ & $1.744 .032,9$ & $-495.832,9$ & $92.000,0$ & $-587.832,9$ \\
\hline Indjolo & $3.379 .200,0$ & $413.833,3$ & $2.965 .366,7$ & $1.415 .618,1$ & $1.549 .748,6$ & $156.000,0$ & $1.393 .748,6$ \\
\hline Air Congo & $3.347 .500,0$ & $324.600,0$ & $3.022 .900,0$ & $1.141 .939,1$ & $1.880 .960,9$ & $219.000,0$ & $1.661 .960,9$ \\
\hline Bongila & $5.405 .000,0$ & $989.333,3$ & $4.415 .666,7$ & $958.413,1$ & $3.457 .253,5$ & $508.000,0$ & $2.949 .253,5$ \\
\hline Moyenne & $2.174 .002,5$ & $287.419,0$ & $1.886 .583,5$ & $1.484 .735,4$ & $401.848,1$ & $167.850,0$ & $233.965,5$ \\
& & & & & & & \\
\hline
\end{tabular}

Sources : Auteurs, enquête fermes modèles pilotes, septembre 2018

Légende : - PB : produit brut ; CI : consommations intermédiaires ; VA : valeur ajoutée ; MOS : main d'œuvre salariée ; RBE : Revenu Brut d'exploitation ; Dot amort : Dotations aux amortissements ; RNE : Revenu Net d'Exploitation.

Le RNE moyen annuel des fermes pilotes modèles est de 233.965,55 CDF soit 146,23 USD. Ce qui représente une faible performance. En milieu paysan congolais, plusieurs spéculations vivrières donnent un RNE annuel de plus de 150 USD (Mpanzu, 2012 ; Oxfam 2017 ; Fuhara, 2018). Il se dégage que seulement quatre fermes sont rentables, leurs résultats nets d'exploitation étant positifs. Il s'agit des fermes Bongila, Air Congo, Indjolo2 et Bafake (Figure 2).

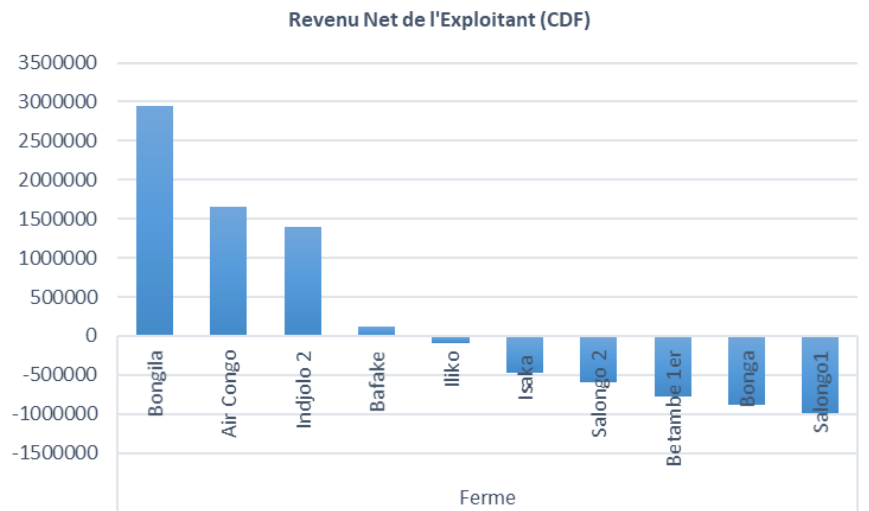

Figure 2.Résultat Net d'exploitation des fermes pilotes modèles du corridor de Monkoto

La ferme Bongila parait la plus organisée de toutes. En effet, avec un RNE de 2.949.253,5 CDF, la rentabilité de cette ferme tient à plusieurs 
facteurs notamment le niveau d'étude du chef de l'exploitation (capital humain), l'ancienneté et le coût de la main d'œuvre. En effet, le responsable de la ferme est de formation de base de technicien en agriculture. Les compétences, le savoir et le savoir-faire acquis par ce fermier augmentent « sa capacité à adopter des nouvelles technologies en réponse aux exigences des cultures et du marché » FAO (2001) cité par Furaha (2018), donc sa capacité productive (Kane, 2010). Bongila est une ancienne ferme. Elle a existé avant 2008 et a reçu plusieurs visites et orientations des animateurs de différents projets de développement mis en œuvre dans la zone, notamment avec le WWF. Le coût de la main d'œuvre y est faible, d'autant plus que les opérations pré culturales ne sont effectuées que pour un ha par saison et principalement pour la culture de maïs. En outre, la main d'œuvre salariée pèse pour moins de $45 \%$ dans la mesure où le fermier utilise les 25 actifs agricoles de son ménage. Cette main-d'œuvre agricole sert aux activités agro-pastorales, à l'entretien des étangs piscicoles, donc à l'augmentation de la production agricole au sens large.

Toutefois, l'incorporation des éléments non payés comme le coût de la main d'œuvre familiale détruit toute la valeur créée par la ferme avec in fine un RNE de -257.723,3 CDF.

Avec un RNE de 1.661.960,9 CDF, la ferme Air Congo figure parmi les plus anciennes et son responsable a une longue expérience et un grand savoir-faire. Elle figure parmi celles qui emploient moins de main d'œuvre salariée soit 53,2\%, juste derrière la ferme de Boangi (44,7\%). Cependant, la prise en compte du coût de la main d'œuvre familiale réduit sensiblement le RNE, soit -78.120 CDF.

Indjolo est une ferme qui figure parmi les plus vastes ( 5 ha mis en valeur). Elle a un RNE annuel de 2.393.748,6 CDF grâce principalement à la culture de riz irrigué ( 2 ha) et la pisciculture. Les autres cultures en dehors du manioc sont faites soit dans la forêt secondaire soit dans la reprise de jachère, ce qui créé une économie sur les charges opérationnelles, notamment la main d'œuvre.

Bafake, est une ancienne ferme qui est en train de se moderniser. Elle doit sa rentabilité de 120.074,5 CDF à l'utilisation de la main d'œuvre familiale et à la vente des fruits étant donné que plusieurs de ses cultures fruitières sont en pleine production. L'ajout du coût de la main d'œuvre aux charges opérationnelles amène le RNE à - 620.468,7 CDF.

Ces résultats issus des analyses traditionnelles de rentabilité en termes de grandeurs comptables viennent compléter et affiner les scores d'inefficacité ainsi dégagés précédemment. Les fermes modèles pilotes les moins rentables économiquement sont aussi les moins efficaces techniquement et donc une amélioration de l'efficacité technique va permettre aux fermes pilotes d'augmenter leur rentabilité. 
L'entrée en production de plusieurs cultures fruitières pourrait améliorer sensiblement les performances tant techniques qu'économiques de ces fermes.

\section{Conclusion}

Cette étude s'est évertuée à analyser les performances de 10 fermes pilotes modèles du corridor de Monkoto. Le modèle agricole mis en place dans ces fermes se caractérise par la diversité des cultures, l’intégration agriculture, élevage et pisciculture ainsi que l'agro foresterie.

Le calcul des grandeurs économiques (PB, VA, RBE, RBN) et la technique d'enveloppement des données (DEA) ont permis de dégager respectivement la rentabilité économique et l'efficacité technique de ces exploitations agricoles. Avec des larges superficies des terres agricoles disponibles et une très faible intensité capitalistique, seulement 4 fermes pilotes sont rentables alors que toutes opèrent en dessous de leur efficacité optimale.

La rentabilité moyenne se situe à 233.965,55 CDF et le score d'efficacité moyen est de 12,7\%. C'est-à-dire que globalement, en prenant l'hypothèse des rendements d'échelle, toutes les fermes ont des grandes marges d'amélioration si les intrants sont utilisés à bon escient. Les fermes pilotes les moins rentables économiquement sont aussi les moins efficaces techniquement et donc une amélioration de la productivité des facteurs de production va permettre aux fermes pilotes d'augmenter leur rentabilité.

Les fermes qui sont rentables sont celles ayant existé avant l'année 2017 et ont déjà procédé à au moins deux récoltes et leurs responsables ont un savoir-faire technologique qui leur confère un avantage tant en termes de combinaison de facteurs de production qu'en termes de planification des activités.

L'amélioration de l'efficacité technique et de la rentabilité économique est une condition pour faciliter la réplication du modèle mis en place par les autres fermiers, créer les opportunités de développement dans la zone et réduire la pression sur la biodiversité. Mais, ceci passerait par un appui technique et financier accru, l'amélioration des infrastructures d'élevage afin de faciliter l'intégration agriculture - élevage et l'amélioration de l'accès aux marchés afin de faire de ces fermes des vrais cadres d'apprentissage pour les milliers de petits agriculteurs de la zone.

\section{References:}

1. ACF, (2008). La pisciculture de subsistance en étangs en Afrique : Manuel technique, $294 \mathrm{p}$.

2. Ale, A-G. (2010). Performances environnementale et socioéconomique des exploitations agricoles au Benin: quelques pistes 
d'innovations pour un développement rural durable en Afrique, ISDA 2010, Montpellier, France, Juin 2010, 10 p.

3. Allou, T-K et Fofana, B. (2018). «Dynamique de l'orpaillage et reconversion socio-économique dans les sous-préfectures de Djékanou et Kokoumbo au centre de la Côte d'Ivoire ", Revue Africaine de Migration et Environnement, vol. 2, n 1\&2, décembre 2018, pp 4975.

4. Amara, N. et Robert, R. (2000). «Mesure de l'efficacité technique : Revue de la littérature», Série Recherche SR.00.07, Département d'économie agroalimentaire et des sciences de la consommation, Université Laval, Québec, pp 16 - 18.

5. Awamia, A. (2014). Efficience technique, allocative et économique des exploitations agricoles de la zone de Souss-Massa, Research gate, https://www.researchgate.net/publication/265550924

6. Azeroual, M. et Doumi, A. (2016). Performances des secteurs de l'agriculture et de l'industrie manufacturière au Maroc: une évaluation par l'approche non paramétrique, Revue Économie, Gestion et Société, N7 juin 2016, ISSN: 2458-6250, 1 - 13 pp.

7. Bessan, E., Alisanto, A. et Tchohntcho, F. (2018). Analyse de l'efficacité productive du riz à Glazoué : évidence empirique à partir du modèle DEA, 14 p.

8. Blancard, S., Boussemart, J-P., Flahaut, J. et Lefer, H-B. (2013). «Les fonctions distances pour évaluer la performance productive d'exploitations agricoles ", Économie rurale [En ligne], 334 | marsavril 2013, mis en ligne le 15 mars 2015, consulté le 09 février 2020.

9. Ceroni, M., Liu S. et Costanza, R. (2012). « Les rôles écologiques et économiques de la biodiversité dans les agro-écosystèmes », Jarvis D.I., Padoch C., et Cooper H.D., Gestion de la biodiversité dans les systèmes agricoles, Bioversity International, Rome, 476-504 pp.

10. Chausse, J-P., T. Kembola et R. Ngonde. (2012). L'agriculture : Pierre Angulaire de l'Economie de la RDC in Herderschee, J., S. D. Mukoko et $\mathrm{T}$. Tshimanga (éditeurs). Résilience d'un Géant Africain : accélérer la Croissance et Promouvoir l'Emploi en République Démocratique du Congo. Vol 2. Etudes Sectorielles. Médiaspaul. Kinshasa. pp 1-97.

11. Culman, H. (1984). Les mécanismes économiques, 2è édition, PUF., Paris, $127 \mathrm{p}$

12. Djimasra N. (2012). Efficacité technique et convergence : l'expérience des pays Africains producteurs de coton, Revue d'Economie Théorique et Appliquée Volume 2 - Numéro 2 - Déc. 2012, pp 177198. 
13. Dugué, P, Vall E, Lecomte, P, Klein, H.D, et Rollins, D. (2004). Evolution des relations entre l'agriculture et l'élevage dans les savanes d'Afrique de l'Ouest et du Centre : Un nouveau cadre d'analyse pour améliorer les modes d'intervention et favoriser les processus d'innovation, Oilseeds and fats, Crops and Lipids, Vol. $11 \mathrm{~N}^{\circ}$ 4/5 Juillet-Octobre 2004, pp 268 - 276.

14. Gafsi, M. et Favreau, JL. (2014), « Diversité des logiques de fonctionnement et durabilité des exploitations en agriculture biologique », Économie rurale, 339-340, pp 128 - 143.

15. Gibon, A, Ryschawy, J, Schaller, N, Blouet A, Coquil, X, Martin P, et al. (2011). L'élevage, un atout pour le développement durable des territoires dans les régions de polyculture-élevage. Rencontres autour des Recherches sur les Ruminants 18 : 369-72. http://www.journees3r.fr/spip.php?article3237

16. Gnimadi, A., (2008). Étude pour l'identification des filières agroindustrielles prioritaires (Bénin).Programme de Restructuration et de Mise à Niveau de l'Industrie des États membres de l'UEMOA (PRMN). UEMOA-ONUDI, 118 p.

17. Fabre, P. (1994). Note méthodologique générale sur l’analyse de filière: utilization de l'analyse filière pour l'analyse économique des politiques. Document de formation pour la planification agricole, FAO, Rome, 105 p.

18. FAO, (2001), Le rôle de l'agriculture dans le développement des pays les moins avancés et leur intégration à l'économique mondiale, Document de travail, Rome, 113 p.

19. Farrell, M.J. (1957). «Measurement of Production Efficiency», Journal of Royal Statistical Society, 120, 253-281.

20. Furaha Mirindi, G. (2017). Analyse comparée des chaines de valeur du riz dans la plaine de la Ruzizi de la Communauté Economique des pays des Grands Lacs (CEPGL) (Thèse de doctorat). Université de Liège Gembloux Agro-Bio Tech, Belgique - 212 pages

21. Hanafi S., Frija A., Jamin J-Y., Zairi A., Hamdane A. et Mailhol J-C. (2015). Les performances des petites exploitations irriguées de la basse vallée de la Medjerda en Tunisie, CahAgric24 : 170-6. 170 - 176 pp, doi :10.1684/agr.2015.0754.

22. Hendrickson, JR., Hanson, JD., Tanaka, DL. et Sassenrath, GF. (2008). Principles of integrated agricultural systems: Introduction to processes and definition. Renewable Agriculture and Food Systems 23 : 265- 71. doi: 10.1017/S174217050700171

23. Hinojos Mendoza, G. (2014). Identification des risques de perte de biodiversité face aux pressions anthropiques et au changement climatique à l'horizon 2100 : Application de la conservation 
dynamique au territoire des Alpes-Maritimes, Thèse de doctorat, Paris Tech, inédit, $328 \mathrm{p}$

24. Huguenin, J-M. (2013). Data Envelopment Analysis (DEA) : Un guide pédagogique à l'intention des décideurs dans le secteur public, IDHEAP - Cahier 278/2013, Chaire Finances publiques, 90 p.

25. IRC. (2018). Analyse des chaines de valeur agricoles dans la plaine de la Ruzizi dans la province du Sud Kivu, Projet IWRM « Maji ya Amani », 209 p.

26. ITAPEL. (2017). Rapport annuel du territoire de Monkoto, 56 p.

27. Kane, G-Q. (2010). Analyse des performances productives des exploitations familiales agricoles de la localité de Zoetele (Cameroun), mémoire de fin d'étude, Université de Yaoundé II, Cameroun, 120 p

28. Levallois, R., Pellerin D. et Perrier J.P. (2014). L'entreprise agricole à l'heure de la gestion stratégique, Agri-Gestion Laval - Economie rurale - F.S.A.A., Université Laval, Québec, 8 p.

29. Minani, B. (2014). Analyse et stratégies de développement de l'agriculture familiale dans un pays post-conflit: Cas de la Province de Kirundo au nord du Burundi. (Thèse de Doctorat en Français). Université de Liège, Faculté Gembloux Agro-BioTech, Belgique, 162 $\mathrm{p}$

30. Miteu, A.R., Sankiana, G., Kinkela, C. et Biloso, A. (2019). Typologie des exploitations agricoles familiales dans les territoires de Kazumba, Dimbelenge et Demba au Kasaï Central en République Démocratique du Congo, Afrique Science 15(1) (2019), pp 377 - 390.

31. Mpanzu, P., Lebailly, P. et Kinkela, C. (2011). Les conditions de production et de mise sur le marché des produits vivriers paysans dans la province du Bas-Congo (R. D. Congo), Les Cahiers de l'Association Tiers-Monde $\mathrm{n}^{\circ}$ 26-20 II, pp $143-150$.

32. Nyemeck, J.B. et Nkamleu G.B. (2006). « Potentiel de Productivité et Efficacité Technique du Secteur Agricole en Afrique », Canadian Journal of Agricultural Economics 54, pp 361-377

33. Oxfam (2017). Recherche Formative - Développement Communautaire dans les provinces de la Sankuru (territoires de Lomela et Kole), du Kasaï central (territoire de Dekese) et de MaïNdombe (territoire d’Oshwe): Périphérie du Parc National de la Salonga, inédit, 185 p

34. Pallière, A. et Rangé, C. (2017). « Contribution de l’approche « système » au dialogue entre techniciens et producteurs. Une expérience dans un projet de développement rural en Guinée ». Communication au colloque Les acteurs de l'aide internationale : vers quels savoirs, engagements et compétences ?, Bordeaux, 11-12 septembre 2017. 
35. Proudlove, N.C. (2000). «Using Excel for Data Envelopment Analysis», Manchester School of Management Working Paper No. 2007, ISBN 186615076 8, 14 p.

36. RDC (2013). Plan national d'investissement agricole (PNIA) 2014 2020, Ministère de l'agriculture et du développement rural, 108 p.

37. RDC (2016). Stratégie et plan d'actions nationaux de la biodiversité (2016 - 2020). Ministère de l'environnement, conservation de la nature et développement durable, 90p.

38. Riolet, M. (2011). Défis de l’analyse de la rentabilité des exploitations agricoles informelles en Afrique du Nord, Afrique Sciences, 23 p.

39. Russelle, MP., Entz, MH., Franzluebbers, AJ. (2007). Reconsidering integrated crop-livestock systems in North America. Agronomy Journal 99 : 325-34. doi: 10.2134/agronj2006.0139

40. Ryschawy, J., Joannon, A. et Gibon, A. (2014). L'exploitation de polyculture-élevage : définitions et questions de recherche. Une revue. Cahiers Agricultures 23 : 346 - 356. doi : 10.1684/agr.2014.0727.

41. Sempore, AW., Andrieu, N., Nacro, HB., Sedogo, MP., Le Gal, PY. (2015). Relevancy and role of whole-farm models in supporting smallholder farmers in planning their agricultural season. Environmental Modelling and Software 68: 147-155. DOI:10.1016/j.envsoft.2015.02.015.

42. Seré, C., Steinfeld H., Groenewold, J. (1996). World Livestock Production systems. Rome : Food and Agriculture Organization of the United

Nations.

ftp://ftp.fao.org/docrep/fao/005/w0027e/w0027e00.pdf

43. Sneessens, I., (2014). La complémentarité entre culture et élevage permet-elle d'améliorer la durabilité des systèmes de production agricole ? Approche par modélisation appliquée aux systèmes de polyculture-élevage ovin allaitant, Thèse de doctorat, Sciences agricoles, Université Blaise Pascal - Clermont-Ferrand II, inédit, 168 p.

44. Soule, B-G., Aboudou, F., Gansari, S., Tassou, M., Yallou, J-D. (2013). Analyse de la structure et la dynamique de la chaine de valeur du manioc au Benin, Lares, Cotonou, Revue 031 - 2013, 75 p.

45. Stessens, J. (2002). Analyse technique et économique des systèmes de production agricole au nord de la Côte d'ivoire, Thèse de doctorat en Sciences Biologiques Appliquées, Katholieke Universiteit Leuven, Faculté des Sciences Biologiques Appliquées, Département Agrotechnique et -Economique, inédit, 302 p

46. Tshomba, J., Nkulu J., Kalambaie, M. et Lebailly, P. (2009). Analyse des effets des Programmes de subventions sur la performance des cultures céréalières (Maïs Zea maysL. et Riz Oriza sp.) en République 
Démocratique du Congo et en Zambie, Revue Africaine d'Environnement et d'Agriculture, 2019 ; 2 (2) ,39-48 pp.

47. Union Européenne (2016). Développement des outils et méthodes d'aide à la décision dans des aires protégées (AP) ciblées par le 11ème FED en République Démocratique du Congo : Etude de cas du Parc national de la Salonga, $103 \mathrm{p}$

48. Vall, E., Koutou, M., Blanchard, M., Coulibaly, K., Diallo, M.A., Andieu, N. (2011). Contribution de l'ntégration agriculture-élevage dans les systèmes agrosylvopastoraux de l'Ouest du Burkina Faso, province du Tuy, Actes du séminaire, novembre 2011, BoboDioulasso, Burkina Faso, 12 p.

49. Wilkins, RJ. (2008). Eco-efficient approaches to land management: a case for increased integration of crop and animal production systems. Philosophical Transactions of the Royal Society B-Biological Sciences 363: 517-25. doi: 10.1098/rstb.2007. 216

50. WWF (2018). Rapport sur l'évaluation du modèle agricole durable des fermes pilotes modèles dans le corridor de Monkoto, 107 p. 